\title{
Study on Risk Factors of Diabetic Nephropathy in Obese Patients with Type 2 Diabetes Mellitus
}

This article was published in the following Dove Press journal:

International Journal of General Medicine

\author{
Fan $\mathrm{Hu}$ (iD \\ Taotao Zhang* \\ School of Public Health, Shanghai \\ University of Traditional Chinese \\ Medicine, Shanghai, People's Republic of \\ China
}

*These authors contributed equally to this work
Purpose: The purpose of this study was to identify diabetic nephropathy risk factors in type 2 diabetes mellitus obese people based on community type 2 diabetes mellitus patients.

Patients and Methods: In the community in Shanghai, we conduct a questionnaire, physical examination, and biochemical examination. The 406 patients included in the analysis were divided into two groups based on whether or not they had diabetic nephropathy. The influencing factors of type 2 diabetes mellitus obese patients were screened by the least absolute shrinkage and selection operator method, and then the influencing factors detected by the least absolute shrinkage and selection operator method were included in the binary logistic regression analysis, and the risk factors for diabetic nephropathy in obese people with type 2 diabetes mellitus were obtained. Finally, the nomogram and forest plot are used to visualize the binary logistic regression results, and the calibration plot and receiveroperating characteristic curve are used to verify the result.

Results: The results showed that family history of diabetes $(\mathrm{OR}=2.091, \mathrm{P}=0.002)$, disease course $(\mathrm{OR}=1.050, \quad \mathrm{P}=0.007)$. hypertension $(\mathrm{OR}=1.768, \mathrm{P}=0.042)$, hyperuricemia $(\mathrm{OR}=2.263, \mathrm{P}=0.003)$, systolic blood pressure $(\mathrm{OR}=1.027, \mathrm{P}<0.001)$, and glycosylated haemoglobin $\mathrm{A} 1 \mathrm{c}(\mathrm{OR}=1.358, \mathrm{P}<0.001)$ were risk factors for diabetic nephropathy.

Conclusion: For obese patients with type 2 diabetes mellitus, they should pay attention to family history of diabetes, disease course and hyperuricemia. Hypertension should be concerned and strictly controlled. Systolic blood pressure and glycosylated haemoglobin A1c will help prolong the survival of diabetic nephropathy patients.

Keywords: obesity, type 2 diabetes mellitus, risk factors, diabetic nephropathy

\section{Introduction}

According to the research, the global prevalence of diabetes mellitus (DM) has reached epidemic proportions and it is expected to increase from 4.51 million in 2017 to an estimated 6.93 million by 2045 . $^{1}$ DM is linked to macrovascular and microvascular complications, which are the leading causes of death and morbidity. ${ }^{2}$ In addition, the risk of death is accompanied by an increase in the number of complications. ${ }^{3}$ Complications of DM increase burden of disease and medical costs. ${ }^{4,5}$ At the same time, DM affects the quality of life of patients and the economic burden on local governments. ${ }^{6}$ Diabetic nephropathy (DN) is one of the important microvascular complications of DM and is common, occurring in $20-40 \%$ of DM patients.

$\mathrm{DN}$ is one of the most common complications of diabetes. ${ }^{7} \mathrm{DN}$ is the leading cause of end-stage renal disease (ESRD) in developed countries. The study predicted that ESRD caused by DN in European countries will increase by $3.2 \%$
School of Public Health, Shanghai

University of Traditional Chinese

Medicine, Shanghai, People's Republic of

China

Tel/Fax +862I5l32246

Email joyking2003@163.com 


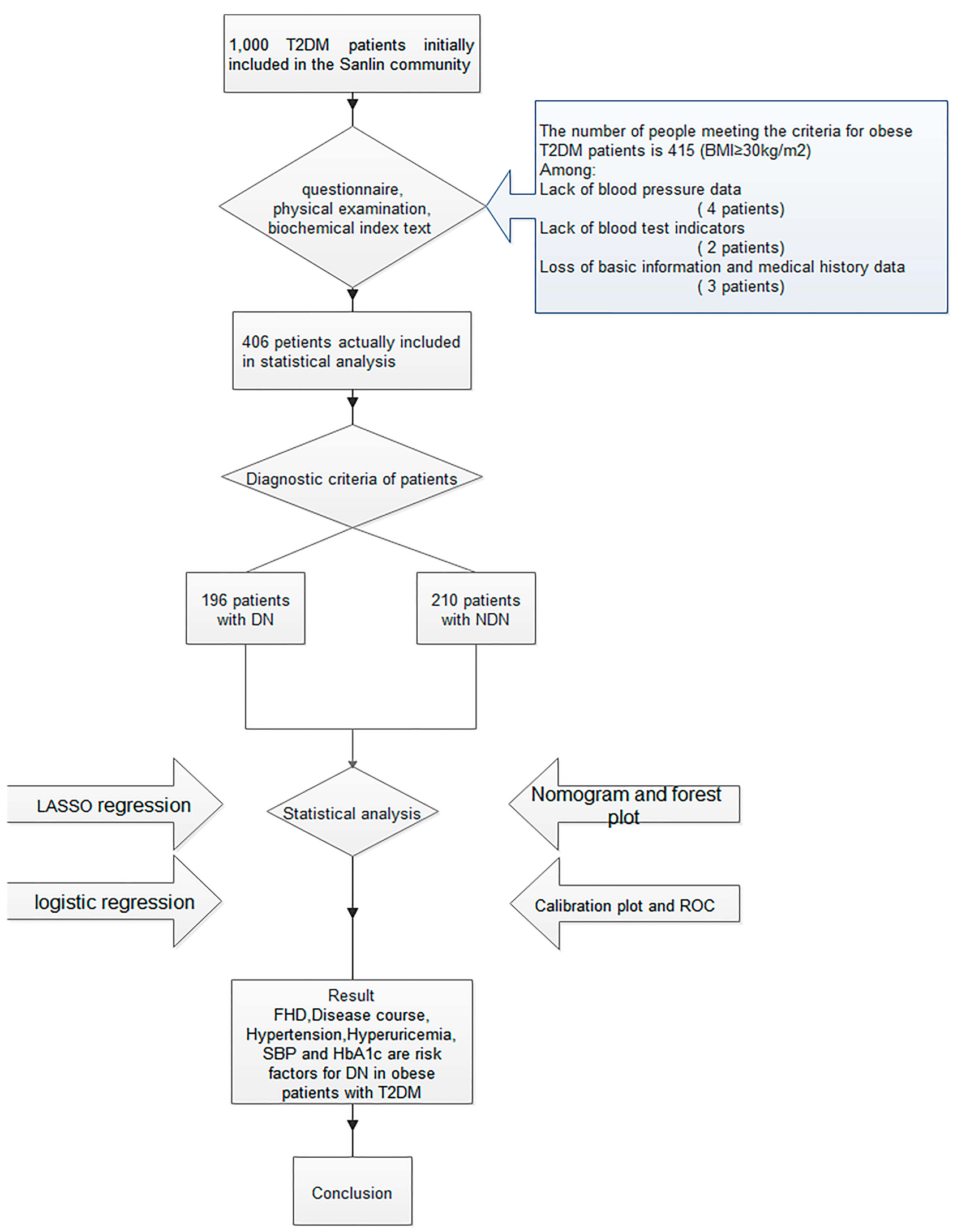

Figure I Logic flow plot of this study.

Note: The logic flow plot shows the entire research process. 
per year between 2012 and $2025 .^{8}$ In China, with the improvement of economic levels and changes in people's lifestyles, diabetes shows an increasing trend every year. According to the latest Diabetes Atlas released by the International Diabetes Federation (IDF) in 2019, the number of diabetic patients in China will reach 1.16 million in 2019, and by 2045 this number will reach 1.47 million. Therefore, it is of great significance to investigate the prevalence of DN and its risk factors. Besides, as people's living standards and quality of life improve, more and more people around them show symptoms of obesity. Therefore, research on obesity is also increasing. Studies have shown that obesity and type 2 diabetes (T2DM) are recognized risk factors for chronic kidney disease worldwide. . $^{9-11}$

In this study, whether to suffer from DN was used as the classification criterion. The purpose of this study was to investigate the prevalence of $\mathrm{DN}$ in obese T2DM patients in the Shanghai community of Sanlin and to analyze the related risk factors of DN in obese T2DM through cross-sectional data, as to provide a theoretical basis for the prevention and treatment of $\mathrm{DN}$ in obese T2DM patients.

\section{Patients and Methods \\ Patients}

We conducted this study in collaboration with the Shanghai University of Traditional Chinese Medicine Affiliated Community Health Center Hospital and Shanghai Community of Sanlin. In this study, we collected 1000 T2DM patients in the Sanlin community. All T2DM patients in the community who participated in the questionnaire. After data processing, the patient data with missing information and incomplete detection indicators were excluded, and patients with $\mathrm{BMI} \geq 30 \mathrm{~kg} / \mathrm{m}^{2}$ were included, finally, 406 valid data were obtained. The 406 participants included in the study were divided into two groups, namely the NDN group $(n=210)$ and the DN group $(n=196)$. Before enrolling the subjects in this study, we received written informed consent from all of them.

\section{Procedure}

We conducted a questionnaire survey, including physical examinations and biochemical tests. With the support of affiliated community health centers and the Central Hospital, T2DM patients in the Sanlin community in Shanghai were investigated. All investigators and researchers participating in the survey were trained and performing well. They were qualified to ensure that procedures are standardized and scientifically rigorous.

The diagnosis of T2DM patients in this study complies with the diagnostic criteria for type 2 diabetes published by the World Health Organization in $1999 .{ }^{12}$ According to the Guidelines for the Prevention and Treatment of T2DM in China, BMI is calculated by dividing body weight $(\mathrm{kg})$ by square height $(\mathrm{m})$. In this study, the patient dressed lightly and measured height and weight using a calibrated spring scale and ruler. According to the World Health Organization (WHO) Obesity Classification Standard, it can be known that body mass index $(\mathrm{BMI}) \geq 30 \mathrm{~kg} / \mathrm{m}^{2}$ is defined as obese. ${ }^{13}$ Obesity is determined by the BMI, which all meet BMI $\geq 30 \mathrm{~kg} / \mathrm{m}^{2}$. Regarding the definition of DN, the study has shown that it can be defined by increased urinary albumin excretion or decreased estimated glomerular filtration rate (e-GFR) or both. ${ }^{14}$ According to the MDND glomerular filtration rate estimation formula, the e-GFR ( $\mathrm{mL} / \mathrm{min})$ was calculated. ${ }^{15}$ In this study, e-GFR was used to define $\mathrm{DN}$, and patients with e-GFR $<60 \mathrm{~mL} / \mathrm{min} / 1.73 \mathrm{~m} 2$ were defined as DN patients. According to strict standards, hyperuricemia is defined by male blood Uric acid (UA) $>420$ umol/L and female blood UA> 360umol/L. ${ }^{16}$ Waistline is the basis for judging whether it is central obesity. Central obesity is defined by male Waistline $\geq 90 \mathrm{~cm}$ and female Waistline $\geq 85 \mathrm{~cm} .{ }^{17}$

In this study, people with a history of hypertension were defined as those with hypertension. In addition, all the physical indicators were measured with standard devices. The patient breathed smoothly, and a soft ruler was used to measure the horizontal circumference of the abdomen at the midpoint of the lower edge of the 12th rib of the anterior superior iliac spine, which is the waist circumference. Systolic blood pressure (SBP) and Diastolic blood pressure (DBP) were measured in standard sitting with OMRON blood pressure monitors. The values of SBP and DBP were measured twice within 5 minutes, and the average of the two was used as the final values of SBP and DBP. ${ }^{18}$ Laboratory indicators measured in this study were fasting blood glucose (FBG), glycosylated haemoglobin A1c (HbA1c), total cholesterol (TC), triglyceride (TG), low-density lipoprotein cholesterol (LDL-C), and high-density lipid protein cholesterol (HDL-C), blood urea nitrogen (BUN), serum creatinine (Scr), UA and e-GFR. In order to obtain blood indicators, all participants were required to fast for at least ten hours and draw venous blood in the morning. Scr is used to calculate 
e-GFR based on the Modification of Diet in Renal Disease Trial with age and gender.

\section{Statistical Analysis}

All the data analysis results of this study were obtained through the software of R software (Version 3.6.2; https:// www.R-project.org). To obtain the risk factors for $\mathrm{DN}$ in obese patients with type 2 diabetes. First, descriptive statistical analysis was performed on the data. The data types in this study belong to measurement data and nonnormally distributed measurement data and counting data. The measurement data are described by the mean and standard deviation ( $\bar{x} \pm \mathrm{s}$ ). For non-normally-distributed measurement data, the median and quartiles [M (P25, P75)] are used. For the count data, it is expressed as a percentage (\%). And then use the least absolute shrinkage and selection operator (LASSO) method to screen the suitable and effective risk factors for obese T2DM patients. LASSO is a method for simplifying highdimensional data. It selects features with non-zero coefficients in the LASSO regression model. Next, the indicators selected in the LASSO regression model were included in the binary logistic regression analysis of risk factors related to diabetic nephropathy, and the odds ratio (OR) value and $95 \%$ confidence interval (CI) were calculated. $\mathrm{P}<0.05$ is statistically significant. Those factors with $\mathrm{P}<0.05$ were significant indicators of $\mathrm{DN}$ in obese patients with T2DM. Finally, using the nomogram and forest plot to visualize the binary logistic regression results, and the Calibration plot and receiver-operating characteristic (ROC) curves are used to verify that the result is reliable.

\section{Results}

A total of 1000 T2DM patients were collected in this study. After data processing, we excluded the patients with missing questionnaire information and incomplete detection indicators, and patients with $\mathrm{BMI} \geq 30 \mathrm{~kg} / \mathrm{m} 2$ were included, finally, we obtained 406 valid data (Figure 1). Among the 931 T2DM patients included in this study, there were 196 DN patients, and the prevalence of DN in obese T2DM patients was $48.28 \%$. In the DN group $(\mathrm{n}=196)$, there were 74 males, accounting for $37.76 \%$, and 122 females, accounting for $62.24 \%$. In the NDN group $(n=210)$, there were 85 males, accounting for $40.48 \%$, and 125 females,
Table I Demographic and Clinical Characteristics of Patients with NDN and DN

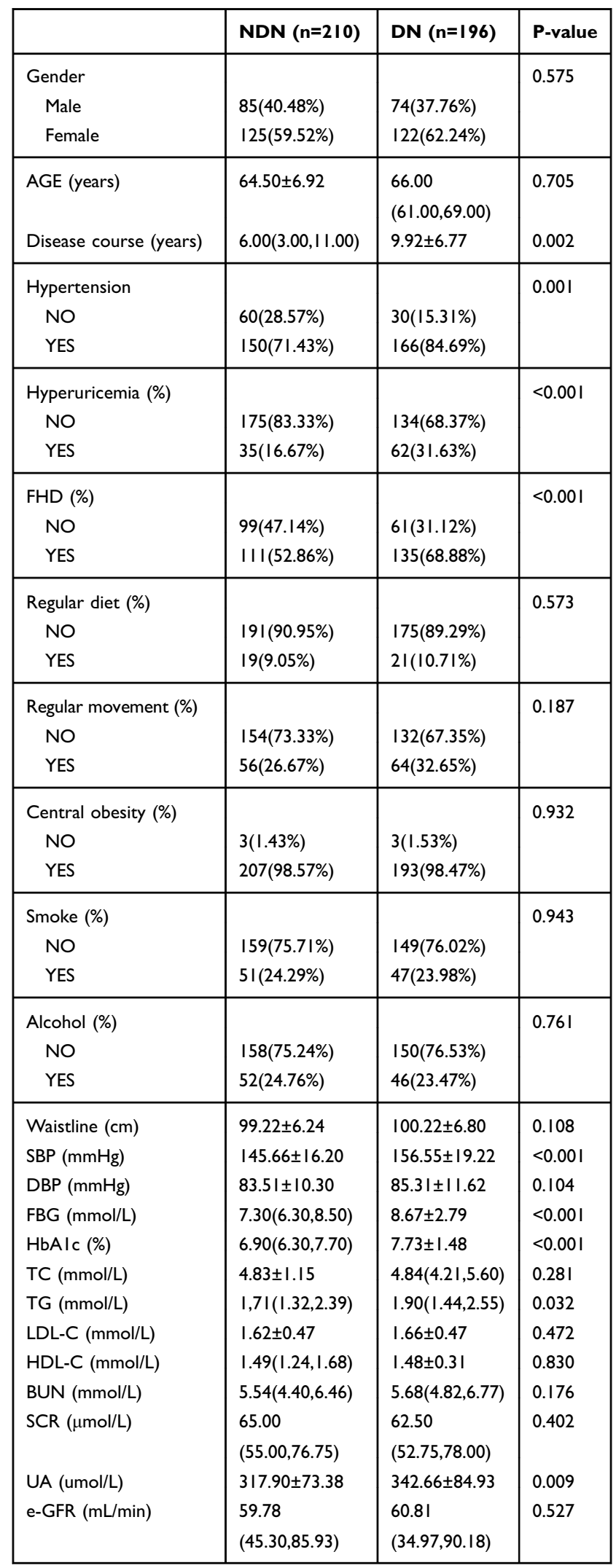


accounting for $59.52 \%$. The concrete demographic and clinical characteristics are given in Table 1.

Based on the analysis of literature search results and questionnaire survey results, from the demographic characteristics index, physical index and biochemical index, 24 potential risk factors were selected and included in the LASSO regression method for analysis (Figure 2A and B). Based on the statistical method of the LASSO regression model, the model type is binomial, and type.measure is deviance (type.measure is used to specify the target parameter that you want to minimize when selecting the model for cross-validation), and non-zero feature variables are selected. So the number of potential variables is reduced from 24 to 5 , including family history of diabetes (FHD), disease course, hypertension, hyperuricemia, SBP, and HbAlc (Table 2). The binary logistic regression analysis was used to analyze the risk factors related to DN in obese T2DM patients. With DN as the dependent variable, the 6 potential risk factors selected in the LASSO regression method were used as independent variables, including FHD, disease course, hypertension, hyperuricemia, SBP, and $\mathrm{HbAlc}$. The result of the binary logistic regression analysis showed that family history of diabetes $(\mathrm{OR}=2.091, \mathrm{P}=0.002)$, disease course $(\mathrm{OR}=1.050, \mathrm{P}=0.007)$. hypertension ( $\mathrm{OR}=1.768, \mathrm{P}=0.042)$, hyperuricemia $(\mathrm{OR}=2.263, \mathrm{P}=0.003)$, systolic blood pressure $(\mathrm{OR}=1.027, \mathrm{P}<0.001)$, and glycosylated haemoglobin $\mathrm{A} 1 \mathrm{c}(\mathrm{OR}=1.358, \mathrm{P}<0.001)$ were risk factors for DN in obese T2DM patients (Table 3). Visualize the results of the binary logistic regression through nomogram. It is based on the six risk factors obtained by the binary logistic regression and can show the incidence of $\mathrm{DN}$ in obese T2DM patients (Figure 3A). The result of the binary logistic regression is shown in the forest plot (Figure 3B). In order to verify that the six risk factors obtained are accurate and credible, calculate the ROC for the binary logistic regression and use the calibration plot to verify. The results obtained are that the area under the ROC curve is 0.75 (Figure 4A), which indicates that the performance is medium, and the calibration plot showed moderate agreement (Figure 4B). In summary, FHD, disease course, hypertension, hyperuricemia, $\mathrm{SBP}$, and $\mathrm{HbA1c}$ are risk factors for DN in obese T2DM patients.

\section{Discussion}

Obesity-related kidney disease is similar to diabetes-related kidney disease in physiology, anatomy, and pathology. ${ }^{19,20}$ Diabetic nephropathy is one of the reasons why patients with chronic kidney disease eventually develop into kidney transplantation. It is clinically manifested by continuous proteinuria and a gradual decrease in the irreversible glomerular filtration rate. The results of this study show that the prevalence of DN in obese T2DM patients in the Shanghai community of Sanlin is $48.28 \%$.

\section{Family History of Diabetes}

In previous studies, it has been pointed out that there is a certain relationship between the genes on the chromosome and T2DM-related kidney disease. ${ }^{21,22}$ In a study on T2DM and DN in the Chinese Han population, it was pointed out that the rs1800783 polymorphism may be a genetic risk factor for diabetic nephropathy. And the family history of diabetes is polymorphic and positively

B
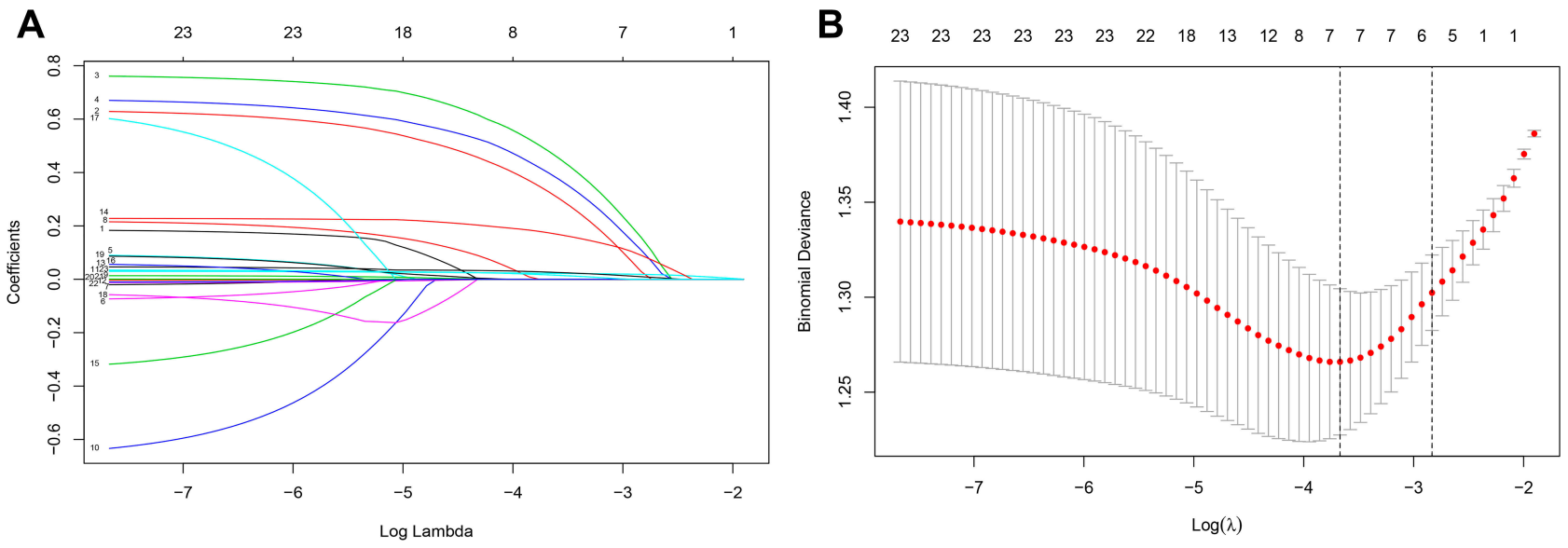

Figure 2 Clinical feature selection using the LASSO regression model.

Notes: (A) Choose the best parameter $(\lambda)$ in the LASSO model and use the lowest standard five-fold cross-validation. By using the minimum standard and the minimum standard of I SE (I-SE standard), a dotted vertical line was drawn at the optimal value. (B) LASSO coefficient profiles of the 6 features. A vertical line was drawn at the value selected using five-fold cross-validation, where the best lambda resulted in 6 features with non-zero coefficients.

Abbreviations: LASSO, least absolute shrinkage and selection operator; SE, standard error. 
Table 2 Coefficients and Lambda.Ise Value of the LASSO Regression

\begin{tabular}{|l|l|l|}
\hline Factors & Coefficients & Lambda.I se \\
\hline Hypertension (\%) & 0.029 & 0.059 \\
Hyperuricemia (\%) & 0.156 & \\
FHD (\%) & 0.109 & \\
SBP (mmHg) & 0.018 & \\
Disease course (years) & 0.010 & \\
HbAlc (\%) & 0.090 & \\
\hline
\end{tabular}

Table 3 Logistic Regression Analysis of DN-Related Risk Factors

\begin{tabular}{|c|c|c|c|c|}
\hline \multirow[t]{2}{*}{ Predictor } & \multirow[t]{2}{*}{ Odds Ratio } & \multicolumn{2}{|c|}{$\begin{array}{l}\text { 95\% Confidence } \\
\text { Interval }\end{array}$} & \multirow[t]{2}{*}{ P-value } \\
\hline & & Lower & Upper & \\
\hline \multirow{2}{*}{$\begin{array}{l}\text { Hypertension } \\
\text { Yes }\end{array}$} & & & & \\
\hline & 1.768 & 1.028 & 3.085 & 0.042 \\
\hline \multirow{2}{*}{$\begin{array}{l}\text { Hyperuricemia } \\
\text { Yes }\end{array}$} & & & & \\
\hline & 2.263 & 1.338 & 3.878 & 0.003 \\
\hline FHD & & & & \\
\hline Yes & 2.091 & 1.329 & 3.319 & 0.002 \\
\hline SBP & 1.027 & 1.014 & 1.041 & $<0.001$ \\
\hline Disease course & 1.358 & 1.150 & 1.614 & $<0.001$ \\
\hline HbAlc & 1.050 & 1.014 & 1.089 & 0.007 \\
\hline
\end{tabular}

correlated with NOS3 rs11771443 in DN, which has not been reported before. ${ }^{23}$ The T2DM obese people are a small part of T2DM and the family history of diabetes is related to the occurrence of diabetes. A study on the clinical and pathological characteristics of diabetic nephropathy and non-diabetic nephropathy pointed out that family history of diabetes can be used as an independent predictor of $\mathrm{DN}$, indicating that there is a relationship between family history of diabetes and the occurrence of DN. Therefore, people with a family history of T2DM among T2DM obese people have a certain relationship with the occurrence of $\mathrm{DN}^{24}$ Our study concluded that family history of T2DM is one of the risk factors for DN in obese T2DM patients.

\section{Disease Course}

A cross-sectional study of T2DM patients in Thailand showed that the course of diabetes is a risk factor for $\mathrm{DN}^{25}$ A national study of 54,670 T2DM patients showed that the course of the disease is closely related to the occurrence of DN. ${ }^{26}$ In a study that examined the relationship between DN and the course of diabetes, it was pointed out that impaired renal function is accompanied by a prolonged course of disease. ${ }^{27}$ The longer the duration of T2DM, the greater the adverse effects on the body, and the same is true for T2DM obese patients. In our study, the disease duration obtained from the data obtained is a risk factor for $\mathrm{DN}$ in obese T2DM patients.

\section{Hypertension}

Studies have now shown that the prevalence of hypertension in T2DM patients is 1.5-3 times the prevalence of non-T2DM populations that match their age. ${ }^{28}$ Hypertension is the basis for the occurrence of arteriosclerosis, which can cause adverse consequences such as endothelial hyperplasia, sclerosis, vascular stenosis and even occlusion. A prospective study investigating T2DM patients in developing countries found that lower e-GFR in patients with hypertension is a major factor leading to the development of diabetic nephropathy, and pointed out that hypertension is an independent risk factor for diabetic nephropathy. ${ }^{29}$ A study based on the Saudi National Diabetes Registry's study of risk factors for $\mathrm{DN}$ in T2DM patients showed that hypertension is one of the most important risk factors for $\mathrm{DN}$ in people with type 2 diabetes. ${ }^{26}$ This study is aimed to explore the risk factors of DN in obese patients with T2DM. Our result confirms that hypertension is a risk factor for DN in obese people with T2DM. This is similar to the results of previous studies, suggesting that hypertension is a risk factor for DN. And our result has important significance for obese people in T2DM patients to pay more attention to the prevention of hypertension.

\section{Hyperuricemia}

Hyperuricemia is closely related to UA. UA is an important indicator to define whether a patient is hyperuricemia. According to strict standards, hyperuricemia is defined by male blood Uric acid (UA) $>420 \mathrm{umol} / \mathrm{L}$ and female blood $\mathrm{UA}>360 \mathrm{umol} / \mathrm{L} .{ }^{16}$ Hyperlipidemia is the main risk factor for DN. ${ }^{30}$ The study has shown that reducing UA levels can improve diabetic nephropathy. ${ }^{31}$ In a study aimed at exploring how hyperuricemia and obesity can cause renal impairment in T2DM patients, it was pointed out that increased obesity and UA increase the risk of kidney damage. ${ }^{32}$ In a study exploring the effect of hyperuricemia on T2DM and DN, it was pointed out that hyperuricemia is a potential risk factor for T2DM and $\mathrm{DN} .{ }^{33}$ It can be known that T2DM obese patients with hyperuricemia are more likely to have DN. In our study, it was proved that 
A

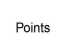

HTN

HUA

FHD

SBP

HbA1C

Disease. Course

Total Points

Risk of DN(Obesity)



YES
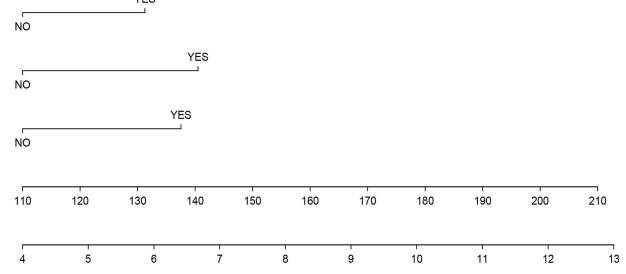

\begin{tabular}{llllllll}
\hline 0 & 1 & 10 & 15 & 20 & 25 & 30 & 35
\end{tabular}

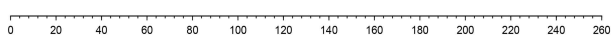

$\begin{array}{lllllllll}0.1 & 0.2 & 0.3 & 0.4 & 0.5 & 0.6 & 0.7 & 0.8 & 0.9\end{array}$
B

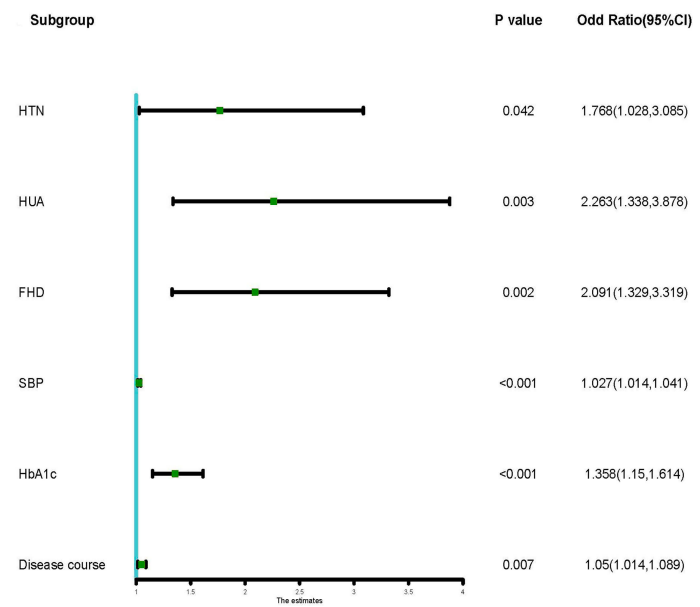

Figure 3 Visual display of risk factors.

Notes: (A) Visualize the risk factors based on binary logistic regression. It can show the incidence of DN in obese T2DM patients. (B) OR forest plot of selected features. Use forest plot to visualize logistic regression analysis.
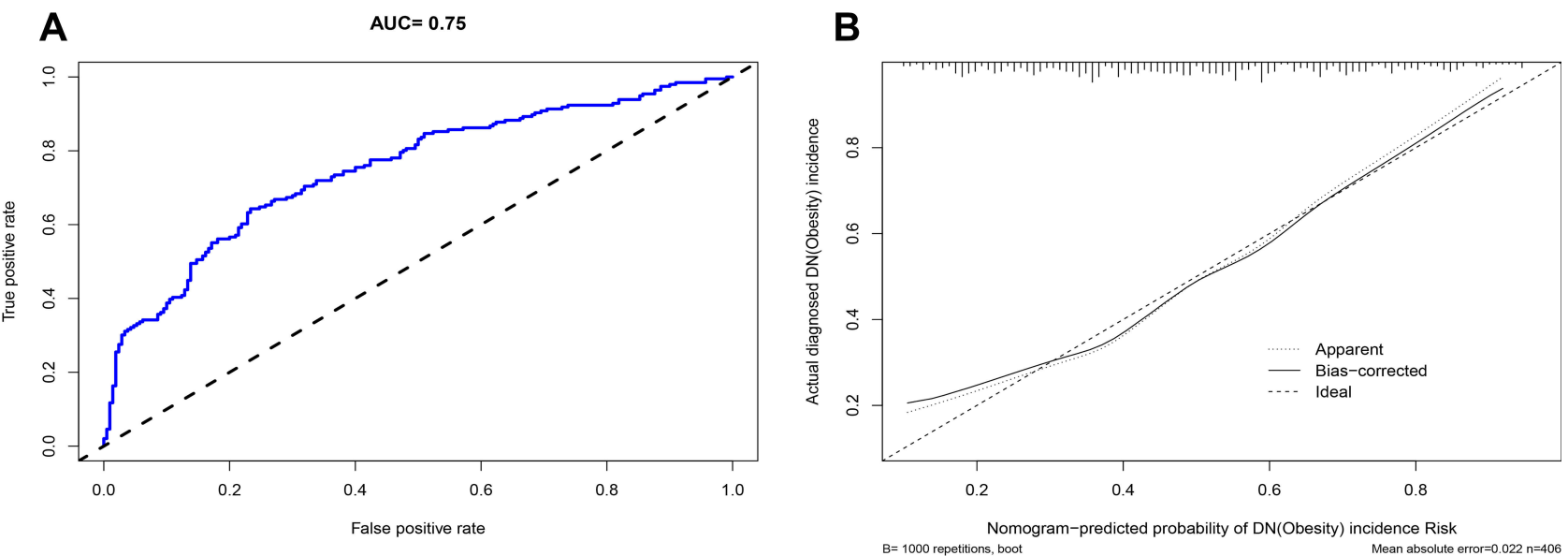

Figure 4 Verification of the binary logistic regression result.

Notes: (A) The blue line represents the performance of the nomogram. (B) The solid line represents the performance of the nomogram and a closer fit to the diagonaldotted line represents a better performance.

hyperuricemia is a risk factor for DN in obese T2DM patients.

\section{Systolic Blood Pressure}

$\mathrm{SBP}$ is one of the main diagnostic indicators of hypertension. The research result has shown that SBP is considered as an independent risk factor for microalbuminuria in T2DM patients, ${ }^{34}$ so SBP can be considered as a risk factor for DN. In an African American study of diabetic nephropathy, blood pressure control was identified as a risk factor for $\mathrm{DN},{ }^{35}$ The results of a prospective diabetes study in the UK showed that strict blood pressure control can significantly reduce the risk of DN. ${ }^{36}$ Similar conclusions have been made in previous studies. ${ }^{37,38}$ In this study on the DN of obese patients with T2DM, we obtained results showing that SBP is one of the risk factors for DN of obese patients with T2DM. As we all know, T2DM obese people are a special group of T2DM people. Based on the results of this study, compared with nonobese people, obese patients with T2DM should also pay attention to the control of SBP.

\section{Haemoglobin Alc}

$\mathrm{HbA} 1 \mathrm{c}$ is a sugar parameter that indicates that the average blood glucose control level at 2 or 3 months is closely related to the risk of diabetes complications. ${ }^{39}$ The results of a study identified the relationship between glycemic control and the development of microalbumin in T2DM 
patients. Statistical analysis of the study also indicated that HbA1c was an independent microalbuminuria risk factor. $^{40}$ Indirectly, it can be explained that $\mathrm{HbA} 1 \mathrm{c}$ is a risk factor for DN. A 10-year follow-up study in Taiwan showed that $\mathrm{HbAlc}$ is an independent predictor of ESDR, that is, HbAlc is used to predict the development of ESRD in T2DM patients. ${ }^{41}$ It is enough to see that $\mathrm{HbA} 1 \mathrm{c}$ is an important factor for $\mathrm{DN}$ in T2DM patients. HbAlc is one of the risk factors in a study on the risk factors of DN in T2DM patients in Shanghai community. ${ }^{42}$ The result in this study indicated that HbAlc is a risk factor for DN in obese patients with T2DM. Combined with the results of this study and comparison with previous results, it is concluded that non-obese T2DM patients still need HbAlc attention. Compared with obese people, HbA1c should be better controlled.

\section{Limitation of This Study}

There are some shortcomings in this study. First, the prevalence of DN in obese patients with type 2 diabetes was screened through a questionnaire. Secondly, the research design of this study is cross-sectional. The exposure variables and the outcome variables of the study coexist, and the time sequence between the exposure and the outcome cannot be judged. Therefore, the causal relationship between the two groups cannot be obtained. In addition, this study is based on the epidemiological investigation of diabetic nephropathy in obese patients with type 2 diabetes. There is a lack of normal weight type 2 diabetic patients as a control and the sample size of the data obtained is limited, and the data has a certain regionality, which is not representative. Therefore, the analysis of risk factors for diabetic nephropathy may have some special features and the lack of some risk factors. The analysis needs to be combined with the actual situation, and it needs to be confirmed and supplemented in the next largescale prospective study.

\section{Conclusion}

This study targeted obese people in T2DM patients. Screen the prevalence of DN in obese patients with T2DM in the Shanghai community of Sanlin. A systematic approach was used to analyze the risk factors of DN in obese T2DM patients. FHD, disease course, hypertension, hyperuricemia, $\mathrm{SBP}$, and HbAlc are all risk factors for DN in obese T2DM patients. This study provides theoretical and clinical basis for preventing and delaying the occurrence and development of diabetic nephropathy in T2DM obese patients. Patients with T2DM obesity should pay attention to the three risk factors of FHD, course of disease and hyperuricemia. For patients with T2DM obesity, early diagnosis and early effective treatment should be adopted. They should always pay close attention to and strictly control blood pressure and HbAlc, which are risk factors that affect the occurrence and development of DN. This approach is expected to extend the survival of patients with DN.

\section{Abbreviations}

DM, diabetes mellitus; DN, diabetic nephropathy; ESRD, end-stage renal disease; IDF, International Diabetes Federation; T2DM, type 2 diabetes mellitus; WHO, the World Health Organization; BMI, body mass index; e-GFR, estimated glomerular filtration rate; UA, uric acid; SBP, systolic blood pressure; DBP, diastolic blood pressure; FBG, fasting blood glucose; HbAlc, glycosylated haemoglobin A1c; TC, Total cholesterol; TG, triglyceride; HDL-C, high-density lipoprotein; LDL-C, low-density lipoprotein; BUN, blood urea nitrogen; Scr, serum creatinine; LASSO, least absolute shrinkage and selection operator, FHD, family history of diabetes; OR, odds ratio; $\mathrm{CI}$, confidence interval; ROC, receiver-operating characteristic.

\section{Ethics Statement}

All participants were carefully informed about the protocol and provided written informed consent before inclusion in the study. The study was approved by the Ethics Committee of Shanghai Oriental Hospital affiliated to Tongji University (Batch number: [2017] Research Review No.20) and performed in accordance with the principles of the Declaration of Helsinki.

\section{Acknowledgments}

The authors thank all the participants and community hospitals that helped to recruit the participants, including Sanlin Community and all participants who volunteered to be studied. Fan $\mathrm{Hu}$ and Taotao Zhang are co-first authors for this study.

\section{Funding}

This research was financially supported by the fourth round of Shanghai Public Health 286 Three-Year Action Plan Key Discipline Construction-Health Education and Health 287 Promotion. (Grant No. 15GWZK1002).

\section{Disclosure}

The authors report no conflicts of interest in this work. 


\section{References}

1. Cho N, Shaw J, Karuranga S, et al. IDF diabetes atlas: global estimates of diabetes prevalence for 2017 and projections for 2045 Diab Res Clin Pract. 2018;138:271-281.

2. care ADAJD. 9. Cardiovascular disease and risk management. Stand Med Care Diab. 2018;41(\(Supplement 1)):S86-S104.

3. Young BA, Lin E, Von Korff M, et al. Diabetes complications severity index and risk of mortality, hospitalization, and healthcare utilization. Am J Managed Care. 2008;14(1):15.

4. Hex N, Bartlett C, Wright D, Taylor M, Varley DJDM. Estimating the current and future costs of Type 1 and Type 2 diabetes in the UK, including direct health costs and indirect societal and productivity costs. Diab Med. 2012;29(7):855-862.

5. Yoon J, Oh I-H, Seo H, et al. Disability-adjusted life years for 313 diseases and injuries: the 2012 Korean burden of disease study. J Korean Med Sci. 2016;31(Suppl 2):S146-S157.

6. Jha V, Garcia-Garcia G, Iseki K, et al. Chronic kidney disease: global dimension and perspectives. Lancet. 2013;382(9888):260-272.

7. Care ADAJD. Microvascular complications and foot care: standards of medical care in diabetes 2019. Diab Care. 2020;43(\(Supplement 1)): S135-S151.

8. Kainz A, Hronsky M, Stel VS, et al. Prediction of prevalence of chronic kidney disease in diabetic patients in countries of the European union up to 2025. Nephrol Dialysis Transpl. 2015;30(suppl_4):iv113iv118.

9. Foster MC, Hwang S-J, Larson MG, et al. Overweight, obesity, and the development of stage 3 CKD: the Framingham Heart Study. Am J Kidney Dis. 2008;52(1):39-48.

10. Hsu C-Y, McCulloch CE, Iribarren C, Darbinian J, Go A. Body mass index and risk for end-stage renal disease. Ann Internal Med. 2006;144(1):21-28.

11. Ejerblad E, Fored CM, Lindblad P, Fryzek J, McLaughlin JK, Nyrén O. Obesity and risk for chronic renal failure. J Am Soc Nephrol. 2006;17(6):1695-1702.

12. Alberti KGMM, Zimmet P. Definition, diagnosis and classification of diabetes mellitus and its complications. Part 1: diagnosis and classification of diabetes mellitus. Prov Rep WHO Consultation. 1998;15(7):539-553.

13. Who, Who, Diet, nutrition and the prevention of chronic diseases introduction. In: Diet, Nut Prev Chronic Dis. 916. Geneva:World Health Organization;2003:1-149

14. de Boer IH, Rue TC, Hall YN, Heagerty PJ, Weiss NS, Himmelfarb JJJ. Temporal trends in the prevalence of diabetic kidney disease in the United States. Jama. 2011;305(24):2532-2539.

15. Huang -Y-Y, Peng C-H, Yang Y-P, et al. Desipramine activated Bcl-2 expression and inhibited lipopolysaccharide-induced apoptosis in hippocampus-derived adult neural stem cells. J Pharmacol Sci. 2007;104(1):61-72.

16. Wise E, Khanna P. The impact of gout guidelines. Current Opinion Rheumatol. 2015;27(3):225-230.

17. Bao Y, Lu J, Wang C, et al. Optimal waist circumference cutoffs for abdominal obesity in Chinese. Atherosclerosis. 2008;201(2):378-384.

18. Whelton PK, Carey RM, Aronow WS, Casey DE, Wright JTJH. 2017 ACC/AHA/AAPA/ABC/ACPM/AGS/APhA/ASH/ASPC/NMA/ PCNA guideline for the prevention, detection, evaluation, and management of high blood pressure in adults. Am College Cardiol. 2017;71(19):213-221.

19. Hill C, Cardwell C, Maxwell A, et al. Obesity and kidney disease in type 1 and 2 diabetes: an analysis of the national diabetes audit. $Q J M$. 2013;106(10):933-942.

20. Hill C, Cardwell C, Patterson C, et al. Chronic kidney disease and diabetes in the national health service: a cross-sectional survey of the UK national diabetes audit. Diab Med. 2014;31(4):448-454.

21. Freedman BI, Colicigno CJ, Sale MM, et al. Replication of a diabetic nephropathy (DN) locus on chromosome 18 in African American (AA) families. $J$ Am Soc Nephrol. 2003;14:291A-291A.
22. Vardarli I, Baier LJ, Hanson RL, et al. Gene for susceptibility to diabetic nephropathy in type 2 diabetes maps to $18 \mathrm{q} 22.3-23$. Kidney Int. 2002;62(6):2176-2183. doi:10.1046/j.1523-1755.2002.00663.x

23. Chen F, Li YM, Yang LQ, Zhong CG, Zhuang ZX. Association of NOS2 and NOS3 gene polymorphisms with susceptibility to Type 2 diabetes mellitus and diabetic nephropathy in the Chinese han population. IUBMB Life. 2016;68(7):516-525.

24. Xu J, Hu XF, Huang W, et al. The clinicopathological characteristics of diabetic nephropathy and non-diabetic renal diseases in diabetic patients. Zhonghua Nei Ke Za Zhi. 2017;56(12):924-929. doi:10.3760/ cma.j.issn.0578-1426.2017.12.007

25. Krairittichai U, Potisat S, Jongsareejit A, Sattaputh C. Prevalence and risk factors of diabetic nephropathy among Thai patients with type 2 diabetes mellitus. J Med Assoc Thai. 2011;94(Suppl 2):S1-5.

26. Al-Rubeaan K, Youssef AM, Subhani SN, et al. Diabetic nephropathy and its risk factors in a society with a type 2 diabetes epidemic: a Saudi national diabetes registry-based study. PloS One. 2014;9:2.

27. Inassi J, Vijayalakshmy R. Role of duration of diabetes in the development of nephropathy in Type 2 diabetic patients. Nat J Med Res. 2013;3(1):000.

28. Arauz-Pacheco C, Parrott MA, Raskin P. The treatment of hypertension in adult patients with diabetes. Diab care. 2002;25(1):134-147.

29. Verma A, Vyas S, Agarwal A, et al. Diabetic kidney disease and hypertension: a true love story. J Clinl Diag Res. 2016;10(3):OC11.

30. Rachmani R, Ravid M. Risk factors for nephropathy in type 2 diabetes mellitus. Compr Ther. 1999;25(67):366-369. doi:10.1007/ BF02944283

31. Momeni A, Shahidi S, Seirafian S, Taheri S, Kheiri S. Effect of allopurinol in decreasing proteinuria in Type 2 diabetic patients. Iran J Kidney Dis. 2010;4(2):128-132.

32. Li Y, Fan $\mathrm{X}, \mathrm{Li} \mathrm{C}$, et al. The relationships among hyperuricemia, body mass index and impaired renal function in type 2 diabetic patients. Endocr J. 2018;65(3):281-290. doi:10.1507/endocrj.EJ170266

33. Shah P, Bjornstad P, Johnson RJ. Hyperuricemia as a potential risk factor for type 2 diabetes and diabetic nephropathy. Jornal Brasileiro De Nefrologia. 2016;38(4):386-387. doi:10.5935/01012800.20160061

34. Rossi MC, Nicolucci A, Pellegrini F, et al. Identifying patients with type 2 diabetes at high risk of microalbuminuria: results of the DEMAND (Developing Education on Microalbuminuria for Awareness of reNal and cardiovascular risk in Diabetes) study. Nephrol Dial Transpl. 2008;23(4):1278-1284.

35. Crook ED, Patel S. Diabetic nephropathy in African-American patients. Current Diab Rep. 2004;4(6):455-461.

36. Adler AI, Stratton IM, Neil HAW, et al. Association of systolic blood pressure with macrovascular and microvascular complications of type 2 diabetes (UKPDS 36): prospective observational study. Bmj. 2000;321(7258):412-419.

37. Ravid M, Brosh D, Ravid-Safran D, Levy Z, Rachmani R. Main risk factors for nephropathy in type 2 diabetes mellitus are plasma cholesterol levels, mean blood pressure, and hyperglycemia. Arch Internal Med. 1998;158(9):998-1004.

38. Park J-Y, Kim H-K, Chung YE, Kim SW, Hong SK, Lee K-U. Incidence and determinants of microalbuminuria in Koreans with type 2 diabetes. Diab Care. 1998;21(4):530-534.

39. Nalysnyk L, Hernandez-Medina M, Krishnarajah GJD. Obesity, metabolism. Glycaemic variability and complications in patients with diabetes mellitus: evidence from a systematic review of the literature. Diab, Obesity Metab. 2010;12(4):288-298.

40. Chen W-Z, Hung -C-C, Wen Y-W, Ning H-C, Gau B-R, Huang -Y-Y. Effect of glycemic control on microalbuminuria development among type 2 diabetes with high-normal albuminuria. Renal Failure. 2014;36(2):171-175. 
41. Yang Y-F, Li T-C, Li C-I, et al. Visit-to-visit glucose variability predicts the development of end-stage renal disease in type 2 diabetes: 10-year follow-up of Taiwan diabetes study. Medicine. 2015;94 (44).
42. Lou J, Jing L, Yang H, et al. Risk factors for diabetic nephropathy complications in community patients with type 2 diabetes mellitus in Shanghai: logistic regression and classification tree model analysis. Int J Health Planning Manage. 2019;34(3):1013-1024.

\section{Publish your work in this journal}

The International Journal of General Medicine is an international, peer-reviewed open-access journal that focuses on general and internal medicine, pathogenesis, epidemiology, diagnosis, monitoring and treatment protocols. The journal is characterized by the rapid reporting of reviews, original research and clinical studies across all disease areas. The manuscript management system is completely online and includes a very quick and fair peer-review system, which is all easy to use. Visit http://www.dovepress.com/ testimonials.php to read real quotes from published authors.

Submit your manuscript here: https://www.dovepress.com/international-journal-of-general-medicine-journal 